Le vin en terre d'Israël d'avant-hier à demain

\title{
Mireille Israël-Lang
}

\section{(2) OpenEdition}

\section{Journals}

Édition électronique

URL : https://journals.openedition.org/geohist/2685

DOI : $10.4000 /$ geohist.2685

ISSN : 2264-2617

Éditeur

Association française de la Revue de géographie historique

\section{Référence électronique}

Mireille Israël-Lang, « Le vin en terre d'Israël d'avant-hier à demain », Revue de géographie historique [En ligne], 19-20 | 2021, mis en ligne le 20 novembre 2021, consulté le 23 novembre 2022. URL : http:// journals.openedition.org/geohist/2685; DOI : https://doi.org/10.4000/geohist.2685

Ce document a été généré automatiquement le 23 novembre 2022.

\section{cc) (ㅇ) $\ominus$}

Creative Commons - Attribution - Pas d'Utilisation Commerciale - Pas de Modification 4.0 International - CC BY-NC-ND 4.0

https://creativecommons.org/licenses/by-nc-nd/4.0/ 


\section{Le vin en terre d'Israël d'avant-hier à demain}

Mireille Israël-Lang

\section{Introduction}

1 La région de la terre d'Israël est celle où les preuves historiques de la culture vitivinicole sont les plus anciennes. Cette zone correspond à toute la Mésopotamie, la rive Est de la Méditerranée jusqu'aux terres de l'Ancienne Egypte (figure 1). 
Figure 1 : Les lieux vinicoles bibliques d'Israël

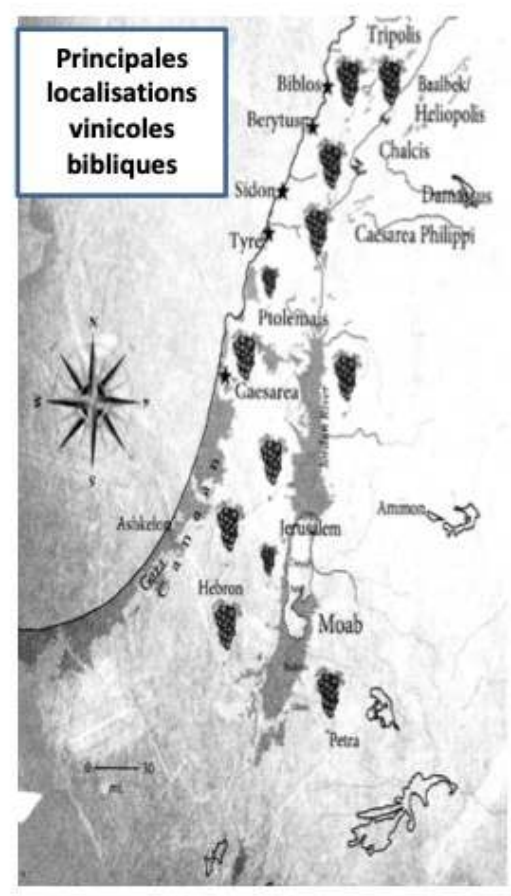

D'après Heskett et Butler, Divine Vintage, Palgrave McMillan, NewYork, Nov. 2012

2 Après la période de l'Antiquité et un temps long avec peu d'évolution, la présence de viticulteurs bordelais dans cette région à la fin du XIX siècle induit un premier renouveau, avec la production d'un vin néanmoins de piètre qualité, souvent comparable à un vin de messe. Près d'un siècle plus tard, les agriculteurs locaux, guidés par les conseils de chercheurs anglo-saxons, aboutissent à une évolution qui sera appelée « la Révolution de la qualité » pour ce vignoble de plus de 5500 hectares. Elle permettra, grâce à l'apparition de vins répondant aux standards qualitatifs internationaux, une reconnaissance progressive mondiale puis la mise en place d'un véritable système économique moderne alliant diversification, amélioration de l'image et rentabilité économiques progressive.

3 L'essor d'un œnotourisme récent, fondé sur des références bibliques constantes, et d'un marketing ciblé vers des groupes religieux juifs et chrétiens d'une part et, d'autre part, vers de nouveaux touristes est avéré. Les Asiatiques (800000 en 2019 d'après le ministère du tourisme israélien), en particulier les Chinois, étaient présents en nombre en Israël avant la pandémie du Covid-19. Précisons que depuis trois ans, le Ministère israélien du tourisme propose des bourses permettant de se rendre durant six mois en Chine pour des guides désireux d'apprendre le chinois.

\section{La permanence de la vigne et du vin dans I'histoire du pays}

4 L'histoire viticole en terre biblique est aussi ancienne que l'installation des habitants. La terre de Canaan était considérée par les Egyptiens comme le pays des vins qui y " coulaient comme des fleuves " ${ }^{1}$. Ils s'y approvisionnaient, comme l'attestent les jarres à vin de la même époque retrouvées dans certaines tombes. La Bible dit que Noé ${ }^{2}$ été 
le premier viticulteur, lui qui, sauvé du Déluge, tenta la culture de la vigne en scellant une alliance avec l'Eternel. On raconte qu'il avait même emmené dans l'Arche des ceps de vigne au même titre que les animaux. Il fut aussi, toujours d'après la Bible, la première personne à s'enivrer (Genèse $9,20-27)$. Alors que le vin voyageait dans l'ensemble $\mathrm{du}$ Moyen-Orient et $\mathrm{du}$ monde méditerranéen, le récit biblique relate qu'Israël, la Terre Promise des Hébreux (Second Livre des Rois,18-32), était couverte de figues et de vignes ${ }^{3}$, produisant du vin en si grande quantité qu'il y en avait davantage que de l'eau. Dans ces mêmes textes, on retrouve de multiples acteurs du monde de la vigne et du vin. La Bible précise encore que les rois d'Israël possédaient de grands vignobles. Le roi David avait même à son service deux employés spécialisés, l'un dans le travail des vignes et l'autre dans la gestion des caves. C'est dans le livre des Nombres (Nb17-23) que l'on trouve le récit le plus fondateur, celui des espions ( «les Explorateurs $")^{4}$ envoyés par Moïse en Terre Promise et qui revinrent avec une grappe de raisin si lourde, qu'installée sur une perche, elle devait être portée par deux hommes, en l'occurrence Josué et Caleb (figure 2).

Figure 2 : Timbre israélien émis le 8 septembre 1954

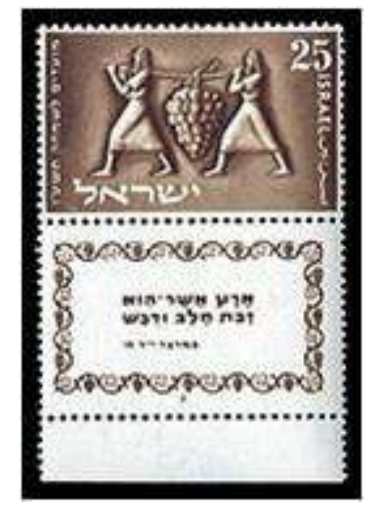

Source : collection personnelle JM Israël

5 Vers -1400 on a pu parler d'un « d'âge d'or » du vin dans la région et les personnes qui se consacraient à la viticulture étaient exemptes de service militaire même en cas d'alerte.

Le renouveau de l'histoire vitivinicole du pays démarre à la toute fin du $\mathrm{XIX}^{\mathrm{e}}$ siècle grâce au baron Edmond de Rothschild ${ }^{5}$, philanthrope juif français, propriétaire de Château Lafite à Bordeaux. Il souhaitait relancer la culture de la vigne dans la région pour donner du travail aux immigrants arrivés d'Europe de l'Est et installés depuis peu (Première Aliyah, de 1881 à 1903) à proximité de Haïfa. C'est avec les cépages bordelais (cabernet sauvignon, merlot, cabernet franc et petit verdot) que la région fut remise en culture sans difficultés majeures, grâce à un climat favorable, humide et semi-tempéré. Rothschild décida même d'aider de nouveaux villages qui plantèrent au Sud de Tel Aviv, puis à proximité de Haïfa, de nombreuses vignes dont les pieds venaient directement de Château Lafite. A partir de la naissance de l'état d'Israël en 1948, parmi les nouveaux immigrants se trouvaient des viticulteurs et des consommateurs intéressés à reprendre le travail de la vigne. C'est en 1957 que fut créé l'Institut israélien du vin, toujours en activité, spécialisé dans la recherche en œnologie et consultant pour tout ce qui concerne la culture du raisin, les espèces et les techniques de production du vin. 
7 Progressivement, à partir des années 1980 et grâce à deux révolutions, le vin israélien a obtenu une reconnaissance à l'International qui l'a dynamisé. C'est en 2007 que Robert Parker, critique de vin américain très influent dans le monde ; a noté pour la première fois les vins israéliens. Il a accordé une note supérieure à 90/100 à 14 vins testés, ce qui, pour lui et les grands acheteurs américains était synonyme de vins de haute qualité. C'est ainsi que cette révolution de la qualité put s'opérer avec l'expertise d'œnologues américains, français et israéliens. Cette transformation fondamentale a démarré par la plantation de nouveaux vignobles, souvent dans des zones d'altitude relativement élevée, comme la région des Monts du Golan entre 400 et 1000 mètres. La production de raisin y était propice grâce à des sols mieux drainés qu'en plaine, et une irrigation minutieuse: le goutte-à-goutte ou la micro-aspersion. La cave des "Golan Heights Winery ", par exemple, a donné comme nom commercial à certaines cuvées l'altitude de culture.

Puis, on a assisté à la révolution des « Boutique wineries », expression californienne des années 1970, venue du français "boutique», pour désigner de petites entreprises viticoles à la recherche d'une production de qualité. Cette deuxième révolution est à l'origine de la multiplication de caves d'un type nouveau (on en dénombre près de 300) qui ont fleuri ces 20 dernières années et qui révèlent le dynamisme de la profession, ouverte sur le monde, pariant sur la qualité et espérant un succès rapide. De plus en plus de vins israéliens (une vingtaine en tous cas) sont connus des critiques du vin et profitent de commentaires encourageants pour réussir leur percée en dehors d'Israël et sont appréciés des connaisseurs, des professionnels et des restaurateurs qui les ajoutent progressivement à leur carte dans la catégorie : "Vins du Monde ».Un autre fait marquant de ces dernières années réside dans le fait que quelques caves israéliennes ont réussi à hisser la qualité de leur production jusqu'à gagner des trophées dans des compétitions internationales à Londres, à Vérone etc. Les "Golan Heights Winery " se sont vues décerner "le trophée de la meilleure cave du monde » alors que «Carmel » a gagné « le Trophée régional et international » décerné à Londres en 2010.

9 En 2013, sur un chiffre d'affaires de 260 millions de dollars, $1 / 5^{\mathrm{e}}$ correspondait aux exportations vers les Etats-Unis, la France, le Royaume-Uni, le Canada, la Pologne et les Pays-Bas. Sur les 300 entreprises vitivinicoles dans le pays, celles du «Top Five» Carmel, Barkan, Golan Heights, Teperberg et Binyamina - réalisent à elles seules, $80 \%$ des ventes. Les autres correspondent pour $98 \%$ à ces «boutique Wineries", petites entreprises produisant à elles toutes 10 à $15 \%$ de la production vitivinicole totale et annuelle israélienne.

\section{Un retour aux sources, mais lesquelles?}

10 Simultanément, la mode du retour à la tradition, aux «temps d'avant » a justifié la nécessité d'un marketing axé sur la Bible. Il s'est développé massivement sur les étiquettes des bouteilles privilégiant par exemple des paysages, des lieux historiques, des noms de personnages, des objets de culte, les sceaux afin d'accentuer l'image d'un vin local, celui de la Terre Sainte. Nous trouvons par exemple :

- Une lampe à huile dont de nombreux exemplaires ont été découverts dans la région et qui pourrait être celle du miracle de Hanoucca (une fiole qui devait contenir la quantité d'huile 
nécessaire à une journée et qui aurait brûlé pendant 8 jours), symbole omniprésent sur les vins des Golan Heights.

- L'utilisation de l'araméen, langue vernaculaire régionale de la période biblique sur les étiquettes des bouteilles de Yatir winery (cave située en plein Néguev).

- L'image du sceau de la tribu de Juda, le lion, sur les mêmes bouteilles (figure 3).

- L'utilisation des versets bibliques concernant Noé dans le hall d'entrée d'un hôtel récent tourné vers l'œnotourisme.

Figure 3 : L'étiquette, le sceau et le texte en araméen sur les bouteilles de Yatir

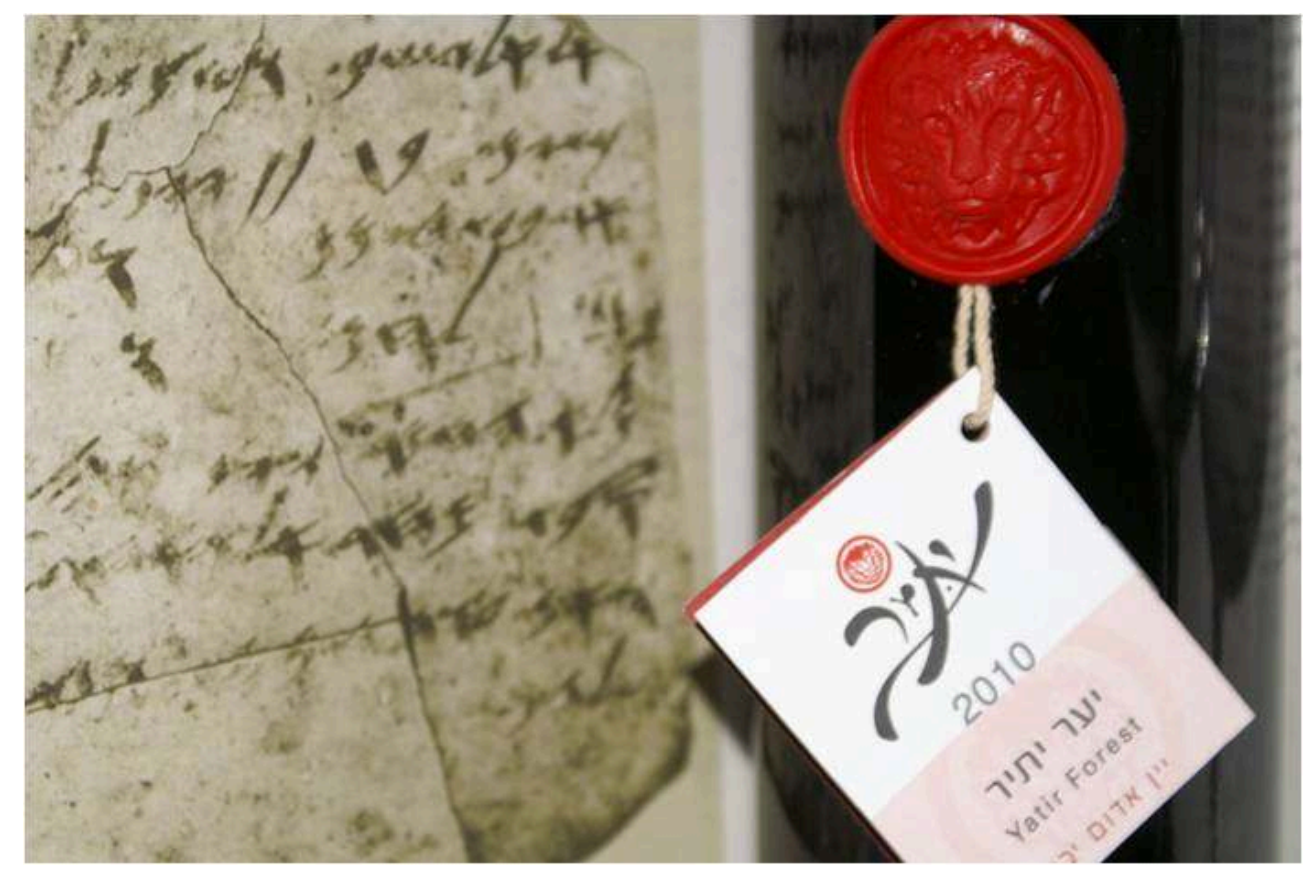

Source : Coffret cadeau Yatir (cliché JM Israël)

11 Par ailleurs certains professionnels cherchent à revenir à des techniques ancestrales, comme cette œnologue viticultrice d'origine géorgienne qui a tenté d'optimiser «la vinification à l'ancienne » en faisant fermenter son vin, après broyage, dans d'énormes amphores d'argile rapportées de Géorgie. C'est sa façon à elle de témoigner, de transmettre un savoir et un savoir-faire ancien.

De plus, une équipe israélienne de chercheurs, archéologues et biologistes, s'attache à reconstituer le plus précisément possible le goût d'un vin de l'Antiquité, sur le modèle du travail du Professeur américain, Patrick McGovern à propos des bières anciennes. Au début 2014, des recherches sur des pépins de raisin antiques ont été publiées et leurs auteurs espéraient qu'elles aboutiraient à la re-création d'une vigne, et donc d'un vin antique. Il semble établi que ce vin cananéen n'ait rien eu à voir avec celui que l'on consomme aujourd'hui, étant proche par exemple des vins romains dont on sait qu'ils étaient consommés fortement épicés pour masquer les goûts liés à leur absence de capacité de conservation.

13 Surtout, un article paru dans le quotidien israélien Haaretz, le 26 octobre 2015, intitulé : « un raisin ancien va-t-il révolutionner l'industrie vitivinicole israélienne?» rend compte d'une nouvelle étape. Il évoque les recherches de l'équipe menée par le Dr Shivi Drori, viticulteur et professeur israélien de biologie et d'œnologie, qui a identifié, grâce au séquençage de l'ADN des pépins de raisins anciens, près de 120 variétés 
différentes de vignes, dont 20 pourraient être utilisées aujourd'hui dans la viticulture. Deux d'entre elles sont uniques (comparables à aucune autre au monde) : Hamdani et Jandali. Or, Recanati a réussi à produire, l'an passé, un vin à partir de raisins blancs. Ces grains utilisés auraient les mêmes caractéristiques que ceux du Hamdani des recherches. Le vin fabriqué en partenariat est le « Recanati's 2014 Marawi » (arôme de pommes et de pêches, agréable à boire, d'après les «happy few "qui l'ont dégusté). Même s'il ne s'agit pour l'instant que d'une étape ( 2480 bouteilles ont été produites), les œnologues israéliens espèrent vraiment aboutir à un vin unique à partir de raisins produits nulle part ailleurs et non plus seulement à des vins d'«inspiration méditerranéenne ». Ce vin a été fièrement présenté à l'« Expo Milan 2015 » comme étant le vin de la Bible.

14 Tous ces exemples prouvent à l'envi qu'il s'agit là, non pas de la recherche d'une réalité, mais plus simplement d'une re-création d'un passé inexistant, mais qui serait porteur de valeurs ancestrales, telles la pureté biblique ou l'intégrité des grands anciens, celles d'un passé idéalisé. Dans certains cas pourtant, c'est le passé réel qui est mis en avant pour assoir la légitimité de l'existence de la cave: on retrouve par exemple sur les bouteilles du vin Tishbi les portraits des ancêtres des propriétaires actuels, vignerons eux aussi dès le tout début du $\mathrm{XX}^{\mathrm{e}}$ siècle.

L'achèvement en cours d'une Route des Vins pensée sur le modèle des routes des vins bourguignonne ou alsacienne, encouragée par les ministères de l'agriculture (pour les vignes proprement dites), du tourisme (pour l'hôtellerie liée à l'œnotourisme ) et de l'industrie (qui prend en charge, de façon spécifique en Israël, les installations des caves situées dans les zones artisanales et non pas, comme en Europe le plus souvent, auprès des vignes) ont pour objectif de faciliter et de développer le tourisme autour de ce vin. Elle a été complétée par une route des vins à vélo qui correspond bien à la demande récente des visiteurs tant nationaux qu'internationaux. C'est un œnotourisme qui est en train de se construire et de se structurer et qui peut, à terme, correspondre à une troisième révolution, œnotouristique cette fois, dans un contexte économique favorable depuis l'éclosion de start-up dans tout le pays au cours des années 1990, au point que le pays ait été surnommé « Nation Start-up » à la parution du célèbre livre de Senor et Sander publié en 2009, devenu un best-seller traduit dans le monde entier. Ces entreprises sont très actives dans les domaines touchant la chimie des sols et du vin, la transition et les économies énergétiques ainsi que l'utilisation de l'eau. Mentionnons pour exemple des entreprises issues de la faculté de physico-chimie du «Technion de Haïfa " (Israel Institute of Technology, université de recherche en sciences et technologies classée parmi les 10 meilleures mondiales).

Récemment ce sont de véritables séjours œnotouristiques qui s'organisent dans des établissements hôteliers haut de gamme qui ont choisi de mettre en relation l'intérêt pour le vin et ses paysages, l'archéologie, la Bible et le bien-être des visiteurs. Séjourner dans l'un de ces hôtels, au milieu des vignes, permet à la clientèle, nationale et internationale d'organiser de véritables circuits de visites de caves, ou de choisir de rester dans l'ambiance "vignes et vin » à l'hôtel, en profitant par exemple de soins de vinothérapie, en fréquentant le bar à vins, en goûtant à une cuisine soucieuse des accords entre les mets et les vins, en faisant des achats viniques à la boutique de l'hôtel.

Des versets de la bible évoquant essentiellement l'histoire de Noé en ce qu'il est considéré comme le premier vigneron, traduits en anglais, font même partie intégrante de la décoration murale des parties communes, comme à l'hôtel Cramim («vignes » en 
hébreu) ouvert il y a une petite dizaine d'années entre Jérusalem et Tel Aviv au cœur de la Judée vitivinicole ainsi que sur les murs des Golan Heights Winery (figure 4).

Figure 4 : Versets bibliques sur les murs de la Winery des Golan Heights

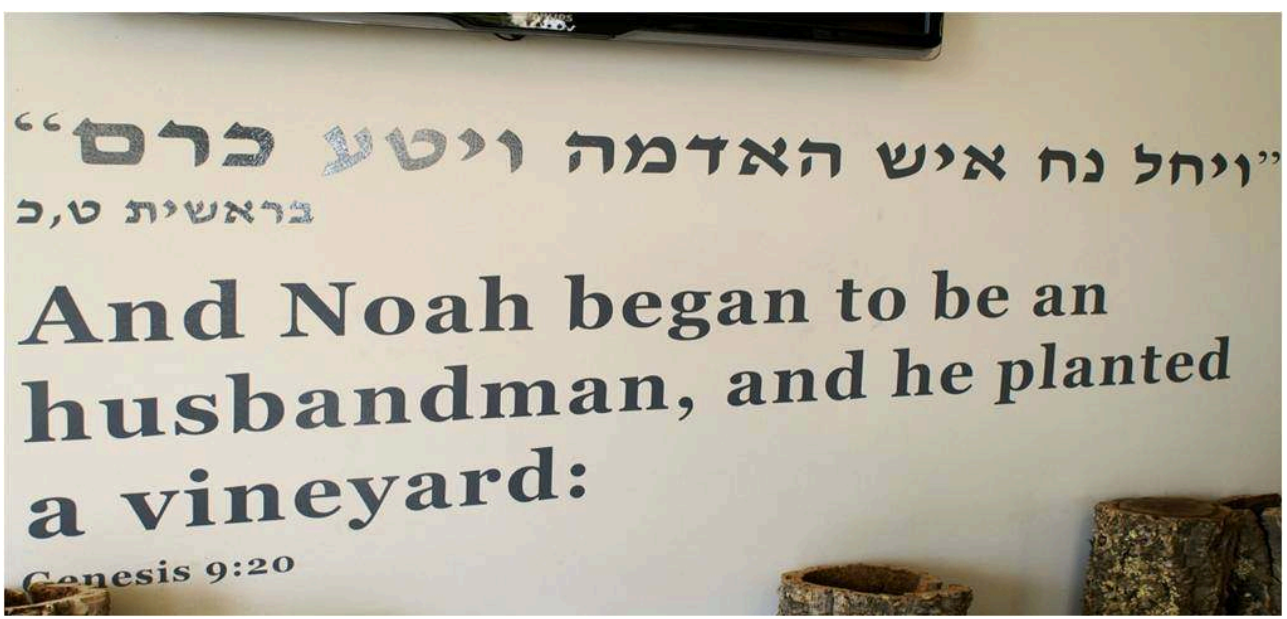

« Noé, d'abord cultivateur, planta une vigne » traduction de la Bible du rabbinat français, édition 2010 Cliché : JM Israel

Le Divin, le Vin, le Tourisme se rencontrent, se complètent et permettent un « va et vient " entre Tradition, Transmission et Modernité. Cette "mise en tourisme» s'organise afin de structurer l'offre en liant le passé, le présent et le futur et permet d'emmener des groupes de touristes nationaux et internationaux dans des zones considérées antérieurement peu intéressantes comme par exemple certaines zones du Neguev sans attractions géologiques et dans lesquelles on retrouve des réservoirs d'eau permettant en partie la création de nouveaux vignobles, des zones périphériques à Jérusalem sans attraits et dans lesquelles se développent aujourd'hui des sites œnotouristiques nouveaux.

\section{Conclusion}

19 Les recherches conjointes d'archéologues, de connaisseurs de la Bible, d'œnologues, de biologistes, associées dans une " mise en patrimoine » unique offerte aux visiteurs, permettent un "va et vient " constant entre la connaissance du passé et la réalité d'Israël actuel. Ces acteurs du monde du vin sont encouragés par tous les acteurs régionaux et soutenus par le Ministère du Tourisme, qui rappelle par son logo l'épisode biblique des Explorateurs (découvrant pour la première fois cette terre qui allait devenir leur) indissociables de l'œnotourisme israélien en développement. Chaque visiteur le voit représenté partout, dès l'arrivée à l'aéroport Ben Gourion de Tel Aviv, jusque dans l'ensemble des lieux touristiques, comme pour assurer la relation entre l'histoire biblique et le présent.

Les glissements continus de la bible au monde actuel sont le témoin d'une transmission aussi bien cultuelle que culturelle d'un savoir, d'un savoir-faire et d'un savoir-être. Ce passage d'un passé biblique antique à une économie œnotouristique contemporaine a conduit à une réussite économique au travers de références liées à la tradition réelle ou fantasmée durant une période moderne aujourd'hui dépassée, puis par une présence 
marketing visant à la capture de nouveaux marchés notamment vers de nouvelles régions et de « Nouveaux Mondes".

\section{BIBLIOGRAPHIE}

Agostini H., Robert Parker. Anatomie d'un mythe, Paris, Seuil, 2007

Argod-Dutard F. (dir), Voyage aux pays du vin. Histoire, anthologie, dictionnaire, Paris, Laffont, 2007

Ben Joseph M., The bible of israeli wines, Ben Shemen, Modan publishing house, 2002

Guide Voir, ISRAËL Jérusalem-Cisjordanie, Paris, Hachette Tourisme, 2010

Heskett P. et Buttler Joël, Divine Vintage. Following the wine from genesis to the Modern Age, New York, Palgrave Macmillan, 2012

Johson H., Pocket Wine book, Londres, Mitchell Beazley, 2013

La Bible, 10è édition, traduit du texte original par les membres du rabbinat français, Paris, Les éditions Colbo, 2010

Sacks E., Goldfischer Y. et Montefiore A., The wine route of Israel, Tel Aviv, Coordinata Publishing House, 2012

Senor D., Singer S., Israël, la nation start-up. Les ressorts économiques du miracle économique israélien, Paris, Maxima, 2011

The Wine Advocate, publication bimensuelle américaine sur le vin du critique Robert M. Parker, titre abrégé en TWA ou WA

Wines of Israël. Mediterranean inspiration, Tel Aviv, The Israel Export and International Cooperation Institute, 2009

Wine, Gourmet et Alcohol, magazine spécialisé israélien (publié en hébreu moderne)

Wine and Gourmet magazine, magazine américain

www.israelvalley.com, site officiel de la Chambre de Commerce France-Israël

www.wines-israel.com, site dédié aux vins israéliens, en anglais

www.wine-israel.co.il, son équivalent, mais cette fois bilingue anglais/hébreu moderne

www.israeli-wine.org, le blog le plus important consacré aux vins israéliens

\section{NOTES}

1. Voir l'inscription de Thoutmosis III gravée sur le temple de Karnak.

2. «Noé le cultivateur commença de planter la vigne. Ayant bu du vin, il fut enivré et se dénuda à l'intérieur de sa tente." 
3. « Un pays de froment et de moût, un pays de pain et de vignobles, un pays d'huile et de miel $2 R$ 18,32 .

4. «C'était l'époque des premiers raisins (...) ils parvinrent au Val d'Eshkol (grappe en hébreu) ; ils y coupèrent un sarment et une grappe de raisin qu'ils emportèrent à deux, sur une perche, ainsi que des grenades et des figues. On nomma ce lieu Vallée d'Eshkol à cause de la grappe qu'avaient coupée les enfants d'Israël » $\mathrm{Nb}$ 17-23.

5. Exposition:» Les Rothschild en France au XIX ${ }^{\mathrm{e}}$ siècle », Paris, BNF, novembre 2012-Février 2013.

\section{RÉSUMÉS}

Israël, dont l'histoire est marquée par la symbolique du vin dès les premiers textes de la Bible, est devenu aujourd'hui, et en quelques années, une terre de viticulture en pleine expansion, grâce au travail de spécialistes ayant fait le choix déterminant de la qualité. Si le vin, dont l'héritage biblique et historique est une notion que nul ne peut remettre en cause et dont les traces sont omniprésentes dans le pays, on ne peut parler d'une véritable viticulture moderne qu'avec l'arrivée d'immigrants et le soutien financier du Baron de Rothschild à la toute fin du XIX ${ }^{\mathrm{e}}$ siècle. Dans les années 1980, deux véritables «révolutions " sont intervenues, accompagnées d'une mutation géographique visible dans le paysage méditerranéen : la révolution de la qualité, grâce à l'expertise d'œnologues français, américains et israéliens, puis la révolution des «Boutique wineries ", synonyme de petites entreprises vitivinicoles à la recherche d'une production de qualité. Le modèle des start-up innovantes dans le domaine de la haute technologie israélienne a marqué le monde du vin qui s'en est inspiré et s'est donné les moyens de la réussite. Les résultats furent très rapides : reconnaissance internationale, diversification de l'offre. L'élaboration d'un tourisme autour du vin s'impose progressivement et une troisième révolution, œnotouristique, cette fois, est en train de réussir.

Israel, whose history is marked by the symbolism of wine from the first texts of the Bible, has become today, and in a few years, a land of wine growing in full expansion, thanks to the work of specialists who have made the decisive choice of quality. If wine, whose biblical and historical heritage is a notion that no one can question and whose traces are omnipresent in the country, one can only speak of a true modern viticulture with the arrival of immigrants and the financial support of Baron de Rothschild at the very end of the 19th century. In the 1980s, two real "revolutions" took place, accompanied by a visible geographical mutation in the Mediterranean landscape: the quality revolution, thanks to the expertise of French, American and Israeli oenologists, and then the revolution of the "boutique wineries", synonymous with small wine companies seeking quality production. The model of innovative start-ups in the field of Israeli high technology has left its mark on the world of wine, which has been inspired by it and has given itself the means to succeed. The results were very quick: international recognition, diversification of the offer. The development of wine tourism is gradually taking hold and a third revolution, this time wine tourism, is succeeding. 
INDEX

Mots-clés : Israël, vin, révolution de la qualité, œnotourisme, Bible

Keywords: Israel, wine, quality revolution, wine tourism, Bible

\section{AUTEUR}

\section{MIREILLE ISRAËL-LANG}

Chercheur associé au réseau international de la Chaire Unesco « Culture et Tradition du vin » de l'Université de Bourgogne

mimisrael@hotmail.com 